\title{
Inverse relationship of Rho kinase and myosin-light chain kinase expression in the aging human detrusor smooth muscle
}

\author{
Timo Kirschstein ${ }^{1^{*}}$, Theresa Sahre ${ }^{1}$, Karoline Kernig $^{2}$, Chris Protzel ${ }^{2}$, Katrin Porath ${ }^{1}$, Rüdiger Köhling ${ }^{1+}$
} and Oliver W. Hakenberg ${ }^{2+}$

\begin{abstract}
Background: Rho kinase (ROCK) and myosin-light chain kinase (MLCK) are key enzymes in smooth muscle contraction. Previous data have suggested that ROCK contribution to human detrusor contraction is increasing with age. Here, we have analyzed the transcriptional expression of Rho kinase isoforms (ROCK1 and ROCK2) as well as MLCK in the aging human detrusor smooth muscle obtained from resected tissue.

Methods: Small pieces of macroscopically healthy human detrusor smooth muscle (urothelium-free) were prepared for quantitative real-time reverse transcriptase polymerase chain reaction (RT-PCR). Transcript expression (mRNA level) of the target genes ROCK1, ROCK2 and MLCK was normalized to three common reference genes (glyceraldehyde-3-phosphate dehydrogenase, $\beta$-actin, phosphoglycerate kinase 1).

Results: We found that across all ages the expression level of ROCK (i.e. ROCK1 and ROCK2 together) was almost equal to that of MLCK in the human bladder. Further, ROCK2 showed a significantly higher expression level than ROCK1. Among all subjects, there was no significant correlation of any single target gene to age, but expression levels of ROCK and MLCK were inversely correlated. Moreover, the within-subject analysis revealed that the ROCK-to-MLCK ratio showed a significantly negative correlation to age. Thus, within a given subject, there is a relative ROCK down-regulation and concomitant MLCK up-regulation.

Conclusions: Together with previous data in human detrusor specimens showing increased ROCK contribution to detrusor contraction, we speculate that the drop of the ROCK-to-MLCK ratio may occur as an attempt to compensate for the increased Rho kinase activity.
\end{abstract}

Keywords: Aging, Detrusor, Human, Real-time RT-PCR, ROCK-to-MLCK ratio

\section{Background}

Age-related changes in urinary bladder contractility are believed to contribute to dysfunctional micturition such as overactive bladder syndrome (OAB). This is a common urological condition associated with urgency, with or without urge incontinence, and increased micturition frequency and nocturia [1]. OAB prevalence increases with age [2], and in order to find new therapeutic strategies for the management of $\mathrm{OAB}$ it is crucial to unravel

\footnotetext{
* Correspondence: timo.kirschstein@uni-rostock.de

${ }^{\dagger}$ Equal contributors

'Oscar Langendorff Institute of Physiology, University of Rostock, Gertrudenstrasse 9, 18057 Rostock, Germany

Full list of author information is available at the end of the article
}

the pathophysiological mechanisms leading to increased bladder contractility. Hence, it is obvious that there is a need for studies on contraction and relaxation mechanisms in the aging human urinary bladder. During the last decade several groups have focussed on the classical adrenergic and cholinergic mechanisms of detrusor motility. For instance, detrusor relaxation following sympathetic $\beta$-adrenoceptor activation was not altered during aging [3], the same was true for cholinergic responses [4]. Although there is no age-related correlation, current medication is still based on antimuscarinics and $\beta$ sympathomimetics. On the other hand, purinergic receptor-mediated contraction increased with age [4] and may thus be regarded as an attractive candidate 
mechanism. Beyond receptor activation, intracellular contraction mechanisms could also be accessible targets for pharmacological manipulation. We have recently addressed the key enzymes Rho kinase (ROCK) and myosin-light chain kinase (MLCK) in the aging human bladder and could demonstrate that ROCK contribution to detrusor contractility was positively correlated with age, while MLCK contribution was not altered during aging [5]. Therefore, we hypothesized that transcriptional up-regulation of Rho kinase could be responsible for this age-related effect. To this end, we performed quantitative real-time reverse transcriptase polymerase chain reaction (RT-PCR) in order to determine the mRNA expression levels of the two isoforms of Rho kinase (ROCK1 and ROCK2) and MLCK in detrusor smooth muscle tissue obtained from patients undergoing cystectomy.

\section{Methods}

\section{Preparation of detrusor smooth muscle strips}

Human detrusor samples were obtained from 41 patients with an average age of $67 \pm 10$ years (mean \pm S.D.; range 46-84 years old; 33 male and 8 female patients, Table 1). These specimens $(1-2 \mathrm{~cm}$ width) were prepared from surgically resected bladder wall obtained from patients who underwent cystectomy. The indication for cystectomy was bladder cancer (urothelial carcinoma) with or without prostate cancer in 35/41 cases, other malignancies with or without infiltration of the urinary bladder in $4 / 41$ cases and neurogenic bladder in 2/41 cases (Table 1). Care was taken to dissect only macroscopically healthy bladder wall tissue for this study, but the anatomical origin could not be standardized due to requirements for routine histopathological examinations especially in radical cystectomy. The specimens always contained urothelium which was removed before small pieces were cut for the PCR analysis. Thus, the tissue used in this study was always obtained from within the detrusor smooth muscle. All in vitro experiments with human material performed in this study were approved by the local ethics committee (University of Rostock), and the informed consent to participate in this study was obtained from each patient.

\section{Quantitative RT-PCR analysis}

Small pieces from the detrusor smooth muscle portion of approx. 1-2 mm width were prepared from the human tissue sample and immediately frozen in liquid nitrogen. Care was taken that these pieces for quantitative PCR were urothelium-free. For mRNA isolation, TRIZOL reagent was used, and total RNA was reverse-transcribed using Moloney murine leukemia virus reverse transcriptase $(200 \mathrm{U} / \mu \mathrm{L})$ and RNasin Plus RNase inhibitor $(40 \mathrm{U} / \mu \mathrm{L}$, both Promega Corporation,
Madison, WI, USA) in the presence of random hexamers $(3 \mu \mathrm{g} / \mu \mathrm{L})$ and dNTP Mix $(10 \mathrm{mmol} / \mathrm{L}$ each, Invitrogen, Carlsbad, CA, USA). For the real-time PCR of the target genes (Rho kinase 1 [ROCK1], Rho kinase 2 [ROCK2], myosin-light chain kinase [MLCK]) as well as three standard reference genes (glyceraldehyde-3-phosphate dehydrogenase [GAPDH], $\beta$-actin [ACTB], phosphoglycerate kinase 1 [PGK1]), we used the QuantiFast SYBR Green PCR Kit (concentration as recommended by the manufacturer, Qiagen Inc., Valencia, CA, USA). The mastermix was aliquoted, $\mathrm{cDNA}$ and primers $(\mathrm{Cf}=20 \mu \mathrm{mol})$ were added. All primers purchased from Molbiol (Berlin, Germany) are given in Table 2. The reference genes ACTB and PGK1 were detected using Qiagen Primer Assays (Qiagen Inc., Valencia, CA, USA). The PCR product length was 156-285 bp (Table 2). Real-time PCR was performed using the ep mastercycler (software realplex 2.2, Eppendorf, Hamburg, Germany) with cycling parameters $95.0{ }^{\circ} \mathrm{C}$ for 2 minutes once, followed by $95.0{ }^{\circ} \mathrm{C}$ for $15 \mathrm{~s}$ and the annealing temperature for $15 \mathrm{~s}$, with normalized fluorescence read at $68.0{ }^{\circ} \mathrm{C}(520 \mathrm{~nm})$ for 40 cycles. The annealing temperatures were adjusted using gradient PCR (ROCK1/GAPDH $53.7^{\circ} \mathrm{C}$, ROCK1/ACTB/PGK1 $57.5^{\circ} \mathrm{C}$, ROCK2/GAPDH $62.6{ }^{\circ} \mathrm{C}$, ROCK2/ACTB/PGK1 $57.5^{\circ} \mathrm{C}$ ). Single product amplification was confirmed by melting curve and gel electrophoresis analysis. Then, the efficiency was determined by PCR using serial dilution of the cDNA. Messenger-RNA (mRNA) expression levels were efficiency-corrected and determined by normalizing the target genes (ROCK1, ROCK2, MLCK) with three standard reference genes (GAPDH, ACTB, PGK1), expressed as the mean of $2^{-\Delta \Delta \mathrm{Ct}} \pm \mathrm{SEM}$.

\section{Statistics}

All data are expressed as means \pm SEM. Statistical comparison was performed using the two-tailed Student's ttest (SigmaStat 3.5). The level of significance is indicated by asterisks $\left({ }^{*} P<0.05 ;{ }^{* *} P<0.01\right)$.

\section{Results}

In the present study, we aimed to investigate the transcriptional expression of Rho kinase (ROCK, isoforms ROCK1 and ROCK2) and myosin-light chain kinase (MLCK) in human detrusor smooth muscle using quantitative RT-PCR, normalized to three commonly used reference genes (glyceraldehyde-3-phosphate dehydrogenase $[\mathrm{GAPDH}], \beta$-actin [ACTB], phosphoglycerate kinase 1 [PGK1]). As it is shown in Fig. 1a, ROCK2 mRNA levels were significantly higher than ROCK1 mRNA levels $(P<0.01$ for all three reference genes, paired two-tailed t-test) indicating that ROCK2 is the predominant Rho kinase isoform in human urinary bladder. The relative mRNA contribution was $15.2 \pm 1.9 \%$ for ROCK1, $42.8 \pm 3.8 \%$ for ROCK2 and $41.9 \pm 4.8 \%$ for 
Table 1 Patient data

\begin{tabular}{|c|c|c|c|c|c|}
\hline No. & Age & Sex & Diagnosis and cystectomy indication & Type of surgery & History \\
\hline 1 & 68 & $\mathrm{~m}$ & Bladder cancer G2 pT2b pN0 (0/9) L0 F0 R0 cM0 & $\begin{array}{l}\text { Radical cystectomy/ } \\
\text { neobladder }\end{array}$ & S/P 1× TUR bladder G3 pT2a \\
\hline 2 & 66 & w & Bladder cancer pT1 G3 pNO LO VO RO MO & $\begin{array}{l}\text { Radical cystectomy/Mainz } \\
\text { Pouch I }\end{array}$ & S/P $1 \times$ TUR bladder G2 pT1 and Cis \\
\hline 3 & 61 & m & $\begin{array}{l}\text { Bladder cancer pT3a pN0 cMx R0 G3 Carcinoma left renal } \\
\text { pelvis pT2 pN3 cMx R1 G3 Prostate cancer pT1a pN0 cMx } \\
\text { Gleason } 2+3=5\end{array}$ & $\begin{array}{l}\text { Radical cystectomy and } \\
\text { nephroureterectomy left/ } \\
\text { ileal conduit }\end{array}$ & $\begin{array}{l}\text { S/P 11× TUR bladder G2 pTa and Cis, at } \\
\text { least pT2 G3 }\end{array}$ \\
\hline 4 & 80 & $\mathrm{~m}$ & $\begin{array}{l}\text { Bladder cancer pT2b (is) pNO cM0 RO LO V0 G3 Prostate } \\
\text { cancer: pT2a pNO (0/13) RO LO V0 Gleason: } 3+3\end{array}$ & $\begin{array}{l}\text { Radical cystectomy/ileal } \\
\text { conduit }\end{array}$ & S/P $1 \times$ TUR bladder G3, pT2a \\
\hline 5 & 68 & m & Bladder cancer pT1 (is) pNo (0/12) G3 R0 L0 V0 & $\begin{array}{l}\text { Radical cystectomy/ileal } \\
\text { conduit }\end{array}$ & S/P $1 \times$ TUR bladder pT1 G3 and pTa G2 \\
\hline 6 & 62 & $\mathrm{~m}$ & Bladder cancer pT2b (is), pNo (0/20) G3 R0 L1 V0 cM0 & $\begin{array}{l}\text { Radical cystectomy/ileal } \\
\text { conduit }\end{array}$ & S/P 1× TUR bladder pT2a G3 \\
\hline 7 & 73 & $\mathrm{~m}$ & $\begin{array}{l}\text { Bladder cancer (with adenoid vegetations) G3 pT1 pNo } \\
\text { (0/11) LO VO RO MO Prostate cancer G2 pT2c PNO LO VO RO } \\
\text { MO Gleason } 3+2=5\end{array}$ & $\begin{array}{l}\text { Radical cystectomy/ileal } \\
\text { conduit }\end{array}$ & $\begin{array}{l}\text { S/P 1× TUR-prostate (no malignancy) S/P 1X } \\
\text { TUR-bladder pT1 G3 }\end{array}$ \\
\hline 8 & 46 & $\mathrm{~m}$ & $\begin{array}{l}\text { Bladder cancer G2 pTa pNO (0/18) LO V0 RO MO and renal } \\
\text { failure (on dialysis) }\end{array}$ & $\begin{array}{l}\text { Radical cystectomy/ileal } \\
\text { conduit }\end{array}$ & $\begin{array}{l}\text { S/P } 1 \times \text { TUR-bladder (necrotic urothelial } \\
\text { carcinoma) }\end{array}$ \\
\hline 9 & 84 & w & $\begin{array}{l}\text { Bladder cancer G4, pT4a pN0 (0/9) M1 (PER) L1 V0 R1 } \\
\text { (with sarcomatoid differentiation) }\end{array}$ & $\begin{array}{l}\text { Radical cystectomy with } \\
\text { cutaneous ureterostomy }\end{array}$ & $\begin{array}{l}\text { S/P } 6 \times \text { TUR-bladder rpTa-1 G1-2, at least G3 } \\
\text { pT2 }\end{array}$ \\
\hline 10 & 50 & $\mathrm{~m}$ & $\begin{array}{l}\text { Prostate cancer } 4+5=9 \text {; pT4 pN1 (8/22) L0 V0 R0 cM0 } \\
\text { (with bladder infiltration) }\end{array}$ & $\begin{array}{l}\text { Radical cystectomy/ileal } \\
\text { conduit }\end{array}$ & $\begin{array}{l}\text { S/P prostate biopsy pT1c Gleason } 5+4=9 \\
\text { and suprapubic catheter after overflow } \\
\text { bladder }\end{array}$ \\
\hline 11 & 60 & $\mathrm{~m}$ & Bladder cancer pT1 pNO cM0 R0 G2 (high grade) & $\begin{array}{l}\text { Radical cystectomy/ileal } \\
\text { conduit }\end{array}$ & S/P $1 \times$ TUR bladder pTa G2 and pT1 G2 \\
\hline 12 & 80 & w & Bladder cancer pT1 pN0 cM0 R0 G2 (high grade) & $\begin{array}{l}\text { Radical cystectomy with } \\
\text { urethrectomy/ileal conduit }\end{array}$ & no history available \\
\hline
\end{tabular}

$1368 \mathrm{~m}$ Neurogenic bladder (contracted bladder) Prostate cancer PT2c RO LO V0; Gleason $3+3=6$

$1474 \mathrm{~m}$ Bladder cancer pT1 pN2 (2/17) G3 RO L1 V0

$1559 \mathrm{~m}$ Bladder cancer pT3b pN2 (2/12) G3 RO L1 V0

$1660 \mathrm{~m}$ Bladder cancer pTis pNO (0/24) RO MO Renal pelvic cancer PT3 G2 RO NO LO VO

Radical cystectomy/ ileal conduit

Radical cystectomy/ileal conduit

Radical cystectomy/ neobladder

Radical cystectomy/ileal conduit and left nephrectomy

$1757 \mathrm{~m}$ Bladder cancer G3 pT1 pNO (0/26) L0 V0 R0 cM0

Radical cystectomy/ neobladder

Radical cystectomy/ileal conduit

Incomplete paraplegia Th4 Polyneuropathy due to alcoholism S/P suprapubic catheter

no history available

S/P 2x TUR-bladder pT1 G2-3 (adenocarcinoma)

S/P 1XTUR-bladder pTis with urothelial carcinoma G2-3 and urothelial carcinoma left renal pelvis G1 pTa

S/P 3x TUR-bladder G3, pTa and mrpTis

S/P 7× TUR-bladder pTa G2 and PT1G3 and rpT1G3

Radical cystectomy/ ileal conduit

Radical cystectomy/ileal conduit

S/P 1× TUR-bladder mpT1 G3 cancer pT2 pNO MO G2

2072 m Bladder cancer pTX pN2 (3/12) G3 R0 cM0

Radical cystectomy/ilea conduit cancer Gleason: 4 + 5 = 9; G3 pT2C pNO LO VO

$2175 \mathrm{~m}$ Bladder cancer G3 pT3b pNo (0/14) L1 V0 R1 cM0 Prostate

$2256 \mathrm{~m}$ Bladder cancer G3 pT3a pN3 L1 V1 pM1 (LYM) Prostate cancer Gleason $2+3=5 ; \mathrm{G} 2$ pT2c

$2377 \mathrm{~m}$ Bladder cancer G3 pT1 pNO (0/13) LO V0 RO cM0 Prostate cancer Gleason $3+4=7$; G3 pT2a pNO LO VO RO MO

2484 m Bladder cancer G3 pT2a pNO (0/17) L0 V0 R0 cM0

2556 w Bladder cancer pT1 (is) pNO cMO RO G3 V0 LO

Radical cystectomy/ileal conduit

Radical cystectomy/ileal conduit

Radical cystectomy/ileal conduit

Radical cystectomy/Mainz Pouch I
S/P TUR-bladder T2a G2-3

S/P 1× TUR-bladder T2 G3, renal failure

S/P TUR-bladder T2 G3

S/P 4X TUR-bladder rpT1G2 and PTa G2

S/P 3x TUR-bladder pTa G1-2 and at least PT2 G3

S/P $1 \times$ TUR-bladder pT1 G3 and mpTis 
Table 1 Patient data (Continued)

\begin{tabular}{|c|c|c|c|}
\hline 26 & 80 & w & Bladder cancer G3 pT1 (is) pNO (0/21) Mx V0 L0 R0 \\
\hline 27 & 55 & w & $\begin{array}{l}\text { Urethra cancer pT2 pNo (0/22) G2 R0 L0 V0 cM0 } \\
\text { (squamous cell carcinoma) }\end{array}$ \\
\hline 28 & 52 & $\mathrm{~m}$ & $\begin{array}{l}\text { Bladder cancer pT3b pN2 (9/20) M1 (lymph) G3 RO L1 V0 } \\
\text { Prostate cancer pT2c pNO cM0 RO L0 VO Gleason } 3+4=7\end{array}$ \\
\hline 29 & 56 & $\mathrm{~m}$ & Bladder cancer pT3a pNO (0/25) G3 RO L1 V0 cM0 \\
\hline 30 & 56 & $\mathrm{~m}$ & $\begin{array}{l}\text { Prostate cancer Gleason } 4 \text { ( } 80 \%)+3=7 \text {; G3 pT4 pN1 (4/ } \\
\text { 12) L1 V0 R1 cM0 (with bladder infiltration) }\end{array}$ \\
\hline 31 & 76 & w & Bladder cancer pT3b pN1 (1/18) G3 R0 L1 V0 cM0 \\
\hline 32 & 70 & $\mathrm{~m}$ & $\begin{array}{l}\text { Bladder cancer G2 pT3b pNo (0/15) MX V0 L0 RO } \\
\text { (squamous cell carcinoma) }\end{array}$ \\
\hline 33 & 81 & m & $\begin{array}{l}\text { Bladder cancer ypTis pNO }(0 / 17) \text { RO cM0 Prostate cancer } \\
\text { pT2a pNO }(0 / 17) \text { RO LO VO, Gleason } 3+3=6\end{array}$ \\
\hline 34 & 63 & $\mathrm{~m}$ & Cancer of prostatic duct \\
\hline 35 & 74 & $\mathrm{~m}$ & Bladder cancer pT1 pNO cM0 RO G2 VO LO \\
\hline 36 & 59 & $\mathrm{~m}$ & $\begin{array}{l}\text { Bladder cancer G3 pT2(is) pN2 (3/25) pM1 (lymph) L1 V1 } \\
\text { RO Prostate adenocarcinoma G2, Gleason } 3+3=6 \text {, pT2b } \\
\text { pNO L0 V0 Pn1 RO }\end{array}$ \\
\hline 37 & 76 & $\mathrm{~m}$ & $\begin{array}{l}\text { Bladder cancer and prostate cancer pT2c pNO (0/18) RO LO } \\
\text { VO pNO, Gleason: } 3+3=6\end{array}$ \\
\hline 38 & 68 & w & Neurogenic bladder, multiple sclerosis \\
\hline 39 & 51 & $\mathrm{~m}$ & Bladder cancer pT4a (is) pNO (0/16) G3 R0 L1 V0 cM0 \\
\hline 40 & 84 & $\mathrm{~m}$ & $\begin{array}{l}\text { Bladder cancer G3 pT3a (is) pNO (0/11) L0 V1 R0 cM0 } \\
\text { Prostate adenocarcinoma Gleason } 4+4=8, \mathrm{G} 3 \text { pT2c pNo } \\
\text { LO Vo R0 cMO }\end{array}$ \\
\hline 41 & 74 & $\mathrm{~m}$ & Bladder cancer G3 pT1 pNO (0/18) L0 V0 RO MO \\
\hline
\end{tabular}

Radical cystectomy/ileal S/P 1× TUR-bladder G3 pT1

conduit

Radical cystectomy/Mainz S/P 1× TUR-urethra (squamous cell

Pouch I

carcinoma pTis)

Radical cystectomy/ileal S/P 1× TUR-bladder G3 pT2

conduit

Radical cystectomy/ S/P 1× TUR-bladder G3 pT2 (is)

neobladder

Radical cystectomy/ileal no history available

conduit

Radical cystectomy/ileal conduit

Radical cystectomy/ileal conduit

Radical cystectomy/ileal conduit

Radical cystectomy/ileal conduit

Radical cystectomy/ neobladder

Radical cystectomy/ neobladder

Radical cystectomy/ileal conduit

Cystectomy/ileal conduit

Radical cystectomy/ileal conduit

Radical cystectomy/ileal

conduit

Radical cystectomy/ileal conduit carcinoma) prostatic duct)

S/P1× TUR-bladder at least PT1 G3

no history available incomplete spastic paraplegia Nicotine abuse

no history available
S/P 1× TUR-bladder pT2 G3 S/P TFS (tissue fixation system - sacrouterine ligament)

S/P 1× TUR-bladder PT2 G3 (squamous cell

S/P 1× TUR-bladder pT1 (m, is) G2-3

S/P mult. TUR-bladder (carcinoma in

S/P1× TUR-bladder mpT2 (is) L1 G2-3

Chronic pelvic pain, detrusor hyperactivity,

S/P 1× TUR-bladder G3, at least PT1 Nx M0

$\mathrm{S} / \mathrm{P} \mathrm{G} 3$, at least $\mathrm{pT} 1$ and carcinoma in situ.

$S / P$ status post, TUR transurethral resection

MLCK (Fig. 1b). When pooling the relative mRNA quantification for ROCK1 and ROCK2, however, there was no consistent difference between ROCK (i.e. ROCK1 and ROCK2 together) and MLCK expression $(P>0.05$ for ACTB and PGK1, $P<0.05$ for GAPDH, unpaired two-tailed t-test) suggesting that ROCK and MLCK transcripts were almost equally abundant (Fig. 1b).

Since we have previously found that Rho kinase (ROCK) contribution to cholinergic detrusor contraction increased with age [5], we hypothesized that expression of this enzyme might be up-regulated in the aging detrusor. In order to test this, we performed a linear correlation analysis between relative mRNA levels and age (Fig. 2a,b). However, over the broad range of ages from 46 to 84 years we could not detect a significant correlation between ROCK1 or ROCK2 to any reference gene used (Fig. 2a,b). The Pearson's correlation coefficients for these bivariate analyses are given in Table 3. With respect to the other key enzyme of smooth muscle contraction, the correlation coefficient between MLCK

Table 2 Forward and reverse primers of ROCK1, ROCK2, MLCK and GAPDH (from Molbiol). ACTB and PGK1 were detected with Qiagen Primer Assays

\begin{tabular}{llll}
\hline Gene name & Forward primer & Reverse primer & PCR product \\
\hline ROCK1 & AAAATTGTGTGAGGAGGACATGG & TTCATCCCAACATTCTTGGATCT & 279 bp \\
ROCK2 & GCAATGCGGTAAAAAGCGA & GGGAATCATGGTGTGACCAA & 217 bp \\
MLCK & GTCTTATGTTATCTTCCATTCTA & TATAATAAACTGTGGCAATACTG & 156 bp \\
GAPDH & AGAAGGCTGGGGCTCATTTG & AGGGGCCATCCACAGTCTTC & 285 bp \\
\hline
\end{tabular}



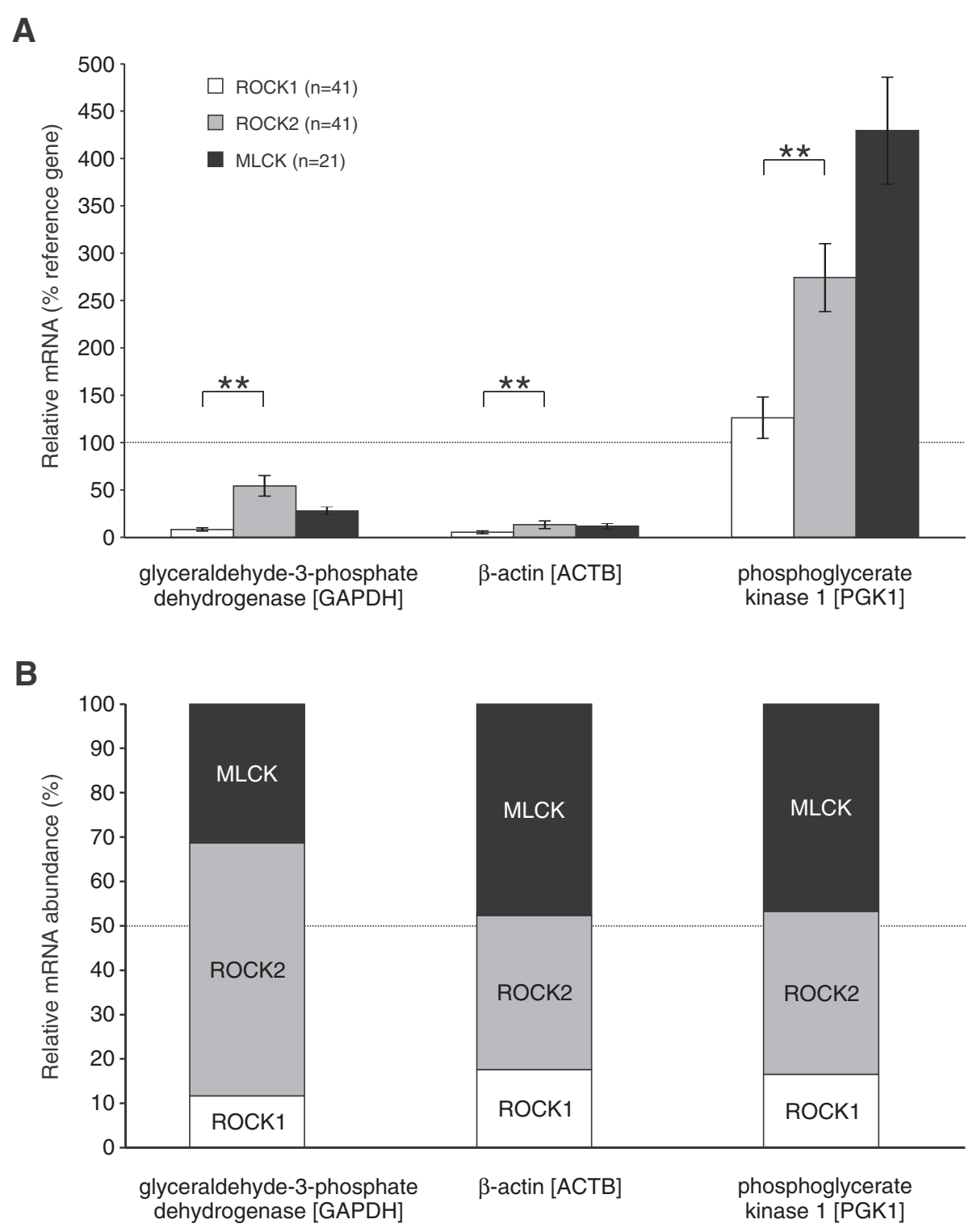

Fig. 1 Expression levels of the contraction enzymes Rho kinase (isoforms ROCK1 and ROCK2) and myosin light-chain kinase (MLCK) in the human detrusor smooth muscle. a Relative mRNA content for target genes ROCK1 (white), ROCK2 (gray) and MLCK (black), expressed as percentage of the reference genes GAPDH, ACTB and PGK1. Note that ROCK2 was significantly more expressed than ROCK1. b Relative mRNA abundance of ROCK1, ROCK2 and MLCK. Note that expression level of ROCK1 and ROCK2 together was similar to that of MLCK

normalized to ACTB and age reached statistical significance (Fig. 3a, Table 3), but it is obvious that MLCK expression levels did not consistently show age-dependent changes. One has to take into account that the vast majority of patients included in this study were cystectomized due to bladder cancer. Thus, mRNA expression of the analyzed contraction enzymes could be altered by the adjacent tumor infiltration. We therefore performed a regression analysis between the tumor size (pathological tumor size, pT1-4 in Table 1) and mRNA expression, but did not obtain significant correlation (data not shown). Since 33/41 (80 \%) patients were male, we tested whether there was a gender specificity in our data. As also shown in Table 3, the correlation coefficients in this subgroup analysis of male patients were similar to those of the total study population. On the other hand, the subgroup analysis for female patients did not reveal statistically significant correlation coefficients either (data not shown).

So far, we have not been able to find robust agedependent differences in mRNA abundance for ROCK1, ROCK2 or MLCK. In these interindividual analyses, however, we have plotted each individual against age and may have missed differential changes of these enzymes 

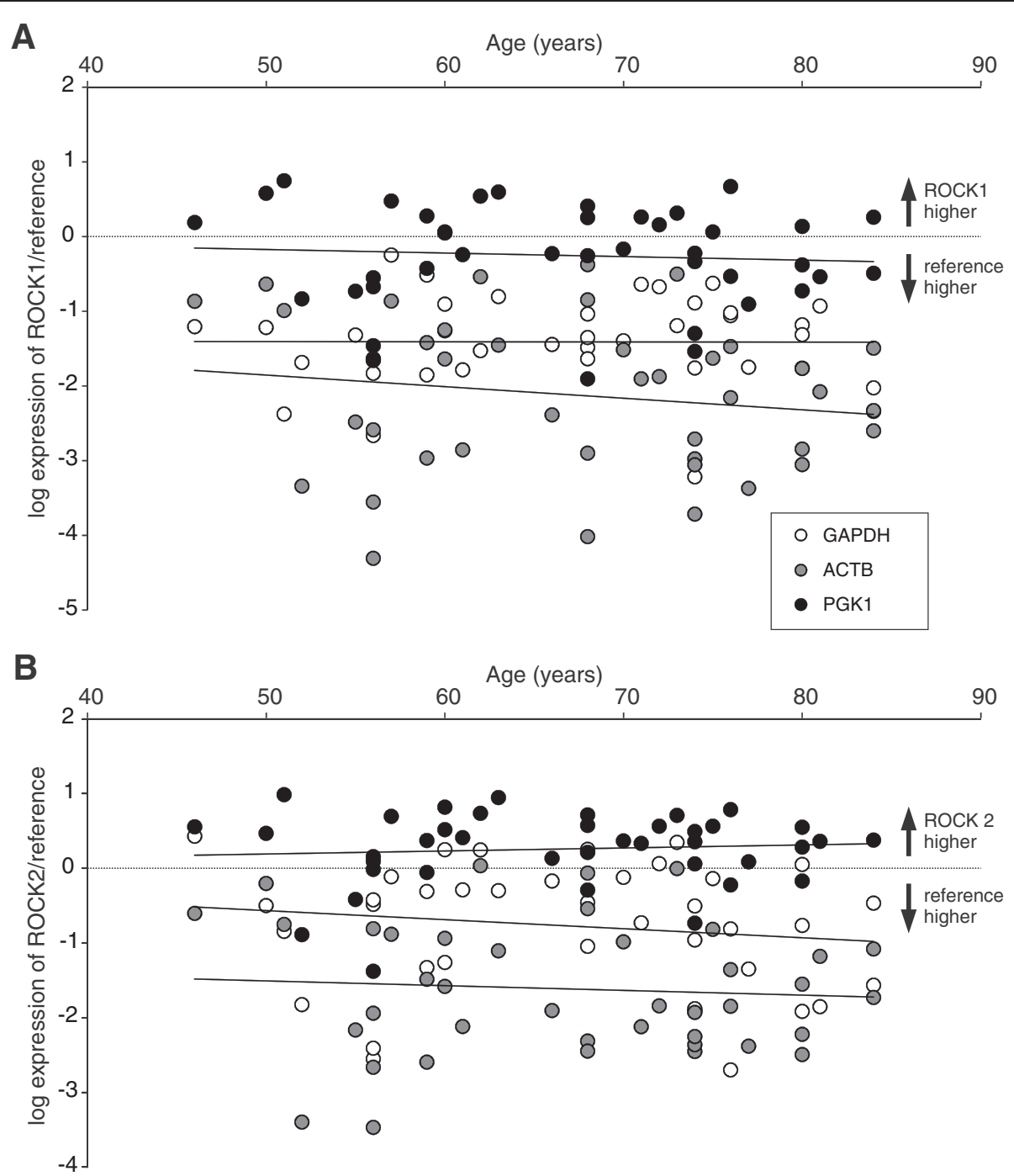

Fig. 2 Expression of ROCK1 and ROCK2 is not correlated with age. Relative mRNA content for the target genes ROCK1 (a) and ROCK2 (b), normalized to reference genes GAPDH, ACTB and PGK1 plotted against the patients' age $(n=41)$. There was no significant correlation between any of these target genes and age

within a given subject. We, therefore, calculated the ROCK-to-MLCK ratio of every subject and plotted this ratio against age. As shown in Fig. 3b, this analysis revealed a decrease of the ROCK-to-MLCK ratio during aging. This negative correlation was significant using all three reference genes (Fig. 3b). Thus, we conclude that there is a robust age-dependent drop of the ROCK-toMLCK ratio in the aging human detrusor smooth muscle. Since ROCK2 was the predominant isoform in human detrusor, we also calculated the correlation coefficients for both isoforms separately (Table 3). These analyses revealed that ROCK2-to-MLCK ratio is consistently inversely related to age, but not the ROCK1-toMLCK ratio (Table 3). These data also suggest that ROCK2 and MLCK are generally negatively correlated. On average, the correlation coefficient between ROCK2 and MLCK mRNA levels was $r=-0.5541(n=21, P<0.01$, two-tailed t-test). In summary, across all subjects we found an inverse relationship between ROCK and MLCK transcription, respectively. Within a given subject, however, aging was associated with a relative ROCK down-regulation and concomitant MLCK up-regulation.

\section{Discussion}

Smooth muscle contraction involves activation of two key enzymes - Rho kinase (ROCK) and myosin lightchain kinase (MLCK). Both have been demonstrated in human detrusor contraction [5], and the present study was performed in order to quantify the mRNA levels for the both isoforms of Rho kinase (ROCK1 and ROCK2) as well as for MLCK in the aging human detrusor smooth muscle. 
Table 3 Pearson's correlation coefficients between target gene (ROCK1, ROCK2 and MLCK, ROCK-to-MLCK ratios) and age using three different reference genes

\begin{tabular}{|c|c|c|c|}
\hline \multirow[t]{2}{*}{ Target gene } & \multicolumn{3}{|c|}{ Reference genes } \\
\hline & GAPDH & ACTB & PGK1 \\
\hline ROCK1 $(n=41)$ & -0.0043 & -0.1584 & -0.0732 \\
\hline only male subjects $(n=33)$ & -0.0375 & -0.1577 & -0.1596 \\
\hline $\operatorname{ROCK} 2(n=41)$ & -0.1436 & -0.0733 & 0.0812 \\
\hline only male subjects $(n=33)$ & -0.0884 & -0.0404 & 0.0810 \\
\hline $\operatorname{MLCK}(n=21)$ & 0.0201 & $-0.4151^{*}$ & 0.0593 \\
\hline only male subjects $(n=18)$ & 0.2899 & -0.3549 & 0.0371 \\
\hline ROCK1-to-MLCK ratio $(n=21)$ & -0.2996 & -0.3325 & $-0.3839^{*}$ \\
\hline only male subjects $(n=18)$ & -0.2802 & $-0.4389^{*}$ & $-0.5364^{*}$ \\
\hline ROCK2-to-MLCK ratio $(n=21)$ & $-0.4757^{*}$ & $-0.4427^{*}$ & $-0.4298^{*}$ \\
\hline only male subjects $(n=18)$ & $-0.4246^{*}$ & $-0.4694^{*}$ & $-0.5854^{* *}$ \\
\hline ROCK-to-MLCK ratio $(n=21)$ & $-0.5297^{* *}$ & $-0.4352^{*}$ & $-0.5181^{* *}$ \\
\hline only male subjects $(n=18)$ & $-0.4625^{*}$ & $-0.4981^{*}$ & $-0.6126^{* *}$ \\
\hline
\end{tabular}

Correlation coefficients in bold indicate that statistical significance was reached using all reference genes $\left({ }^{*} P<0.05,{ }^{* *} P<0.01\right.$; t-test $)$

Quantified PCR data do necessarily depend on the choice of reference genes used for normalization. In particular, altered expression of a reference gene is a potential pitfall in PCR studies that may substantially contaminate the quantification of the genes of interest. With respect to the present study, selection of reference genes in human bladder tissue primarily derived from bladder cancer patients is not simple [6]. Although we have used macroscopically tumor-free tisse specimens, reference genes could be altered by the adjacent pathology. First, we have used the most commonly used reference gene, glyceraldehyde-3-phosphate dehydrogenase (GAPDH). This, however, may be up-regulated in bladder cancer [7]. Hence, we also used $\beta$-actin (ACTB) which was not significantly altered in bladder cancer [6] or prostate cancer [8]. The last reference gene, the glycolysis enzyme phosphoglycerate kinase 1 (PGK1), is a target gene of both the myc oncogene pathway and the hypoxia inducible factor $1 \alpha$ (HIF-1 $\alpha)$ and is therefore considered as a marker gene for a number of malignant tissues such as kidney cancer [9] or colon cancer [10]. Recently, HIF-1 $\alpha$ activation and subsequent PGK1 upregulation has also been demonstrated in bladder cancer [11]. Taken together, cancer-associated up-regulation of the reference genes used in the present study was (i) definitely present (PGK1), (ii) vaguely present (GAPDH) or (ii) absent (ACTB). As a consequence, altered target gene expression was only accepted to be relevant when it could consistently be observed with all three reference genes.

Obviously, the target genes may also be altered by the malignant pathology present in the vast majority of cases. While care was taken to dissect tissue from the macroscopically healthy bladder wall with non-infiltrated urothelium, we cannot exclude the enzyme expression to be altered in detrusor tissue from bladder cancer patients. At least, the non-selective ROCK inhibitor HA1077 has been found to be beneficial against urothelial cancer [12] and this finding might be interpreted as an indication for an enhanced expression of this enzyme in bladder cancer. However, we did not observe a significant correlation between tumor size and ROCK1 or ROCK2 mRNA expression.

First, our data demonstrated that transcript levels for ROCK (i.e. ROCK1 and ROCK2 together) were similar to that for MLCK. Hence on the transcriptional level, both key enzymes were equally expressed. This is important to note, since it is consistent with functional data from cholinergic contractions of human detrusor specimens that showed an equal pharmacological effect of ROCK and MLCK inhibition, respectively [5]. Thus, both enzymes are not only equally transcribed, but also equally involved on the functional level. Moreover, the present study has also shown that ROCK2 mRNA is significantly more abundant than ROCK1 mRNA. Hence, the predominant Rho kinase isoform in the human detrusor is ROCK2. This is in contrast to rat detrusor tissue, which showed equal transcript levels for ROCK1 and ROCK2 [13]. The predominance of ROCK2 in the human detrusor is a clinically relevant finding, since Rho kinase inhibitors are currently developed for different potential indications, and research so far has largely been concentrated on the ROCK1 isoform - in particular in vascular smooth muscle. The notion that this isoform is implicated in arterial hypertension [14] has launched substantial pharmaceutical interest in developing ROCK1 inhibitors as a new class of antihypertensive medication [15]. Moreover, increased ROCK function was also found to be involved in pulmonary hypertension, and again transcription levels of ROCK1 were more markedly enhanced than transcription levels of ROCK2 in both rat [16] and human [17]. Currently, specific ROCK2 inhibitors are not available, but the present study suggests that ROCK 2 might be regarded as potential therapeutic target in overactive bladder syndrome.

Another major finding was that the ROCK-to-MLCK ratio showed a significant negative correlation with aging, even though there was no correlation between any of these genes and age. In other words, within a given subject, there was a relative ROCK downregulation and - at the same time - MLCK upregulation. This was clearly an unexpected finding, since ROCK contribution to contraction increased with aging as opposed to the contribution of MLCK [5]. Given the fact that the down-regulation of ROCK in the aged detrusor was primarily due to down-regulation of ROCK2, 

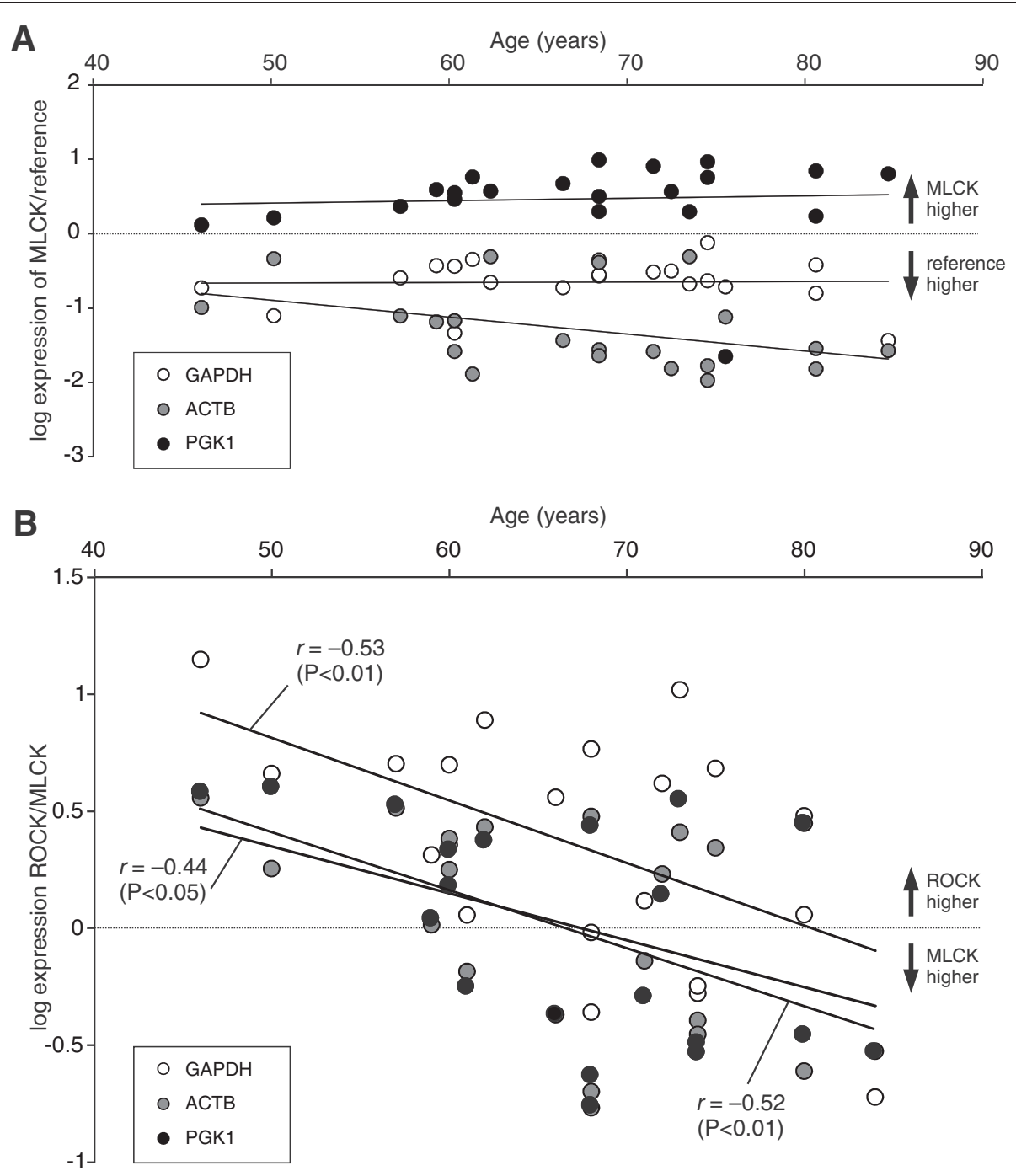

Fig. 3 The ROCK-to-MLCK ratio is negatively correlated with age. a Relative mRNA content for the target gene MLCK, normalized to reference genes GAPDH, ACTB and PGK1 plotted against the patients' age $(n=21)$. There was no consistent correlation between MLCK and age. $\mathbf{b}$ Ratio of ROCK expression to MLCK expression, plotted gainst age $(n=21)$. Note that there was a significant negative correlation between the ROCK-to-MLCK ratio and age using all three reference genes

one can speculate about the mechanism of this agedependent transcriptional alteration. We already know from rodent studies that Rho kinase is involved in bladder hyperactivity [18], but in addition, it was always the ROCK1 isoform that was identified to be up-regulated in rat decompensated detrusor hypertrophy [19] or in altered detrusor contractility of diabetic rabbits [20]. Thus, down-regulation of ROCK 2 in aged human detrusor tissue is therefore a rather uncommon example of transcription regulation, and possibly not the primary cause. It is therefore intriguing to speculate whether transcriptional ROCK2 down-regulation might be secondary in order to compensate for an enhanced Rho kinase function in the aging bladder. Further studies are needed to address this question experimentally.

\section{Conclusion}

The present study shows that ROCK and MLCK are inversely regulated and that down-regulation of ROCK and in particular ROCK2 - as occurs during aging is counterbalanced by an up-regulation of MLCK. In conclusion, we suggest that there is a physiological homeostatic regulation of both enzymes which is probably disturbed in the aged detrusor. Therefore, pharmacological intervention such as specific ROCK2 inhibitors might be beneficial to reduce bladder overactivity in the elderly.

\section{Abbreviations}

ACTB: $\beta$-actin; GAPDH: Glyceraldehyde-3-phosphate dehydrogenase; MLCK: Myosin-light chain kinase; mRNA: Messenger-RNA; 
PGK1: Phosphoglycerate kinase 1; ROCK: Rho kinase; RT-PCR: Real-time reverse transcriptase polymerase chain reaction.

\section{Competing interests}

The authors declare that they have no competing interests.

\section{Authors' contributions}

TK designed the study, performed the statistical analysis, and prepared the manuscript. TS and KP carried out the molecular genetic studies. KK performed analysis and prepared the manuscript. CP and OWH provided the human bladder specimens. RK and OWH conceived of the study, and participated in its design and coordination and prepared the manuscript. All authors read and approved the final manuscript.

\section{Acknowledgements}

The authors wish to thank Tina Sellmann, Hanka Schmidt and Bernd Memmener for excellent technical assistance. This work was supported by a grant from the Medical Faculty of the University of Rostock (FORUN program).

\section{Author details}

'Oscar Langendorff Institute of Physiology, University of Rostock, Gertrudenstrasse 9, 18057 Rostock, Germany. 'Department of Urology, University of Rostock, Rostock, Germany.

\section{Received: 20 February 2015 Accepted: 5 October 2015}

\section{Published online: 15 October 2015}

\section{References}

1. Abrams P, Cardozo L, Fall M, Griffiths D, Rosier P, Ulmsten U, et al. Standardisation Sub-Committee of the International Continence Society. The standardisation of terminology in lower urinary tract function: report from the standardisation sub-committee of the International Continence Society. Urology. 2003;61:37-49.

2. Milsom I, Abrams P, Cardozo L, Roberts RG, Thüroff J, Wein AJ. How widespread are the symptoms of an overactive bladder and how are they managed? A population-based prevalence study. BJU Int. 2001;87:760-6.

3. Schneider T, Fetscher C, Michel MC. Human urinary bladder strip relaxation by the $\beta$-adrenoceptor agonist isoprenaline: methodological considerations and effects of gender and age. Front Pharmacol. 2011;2:11.

4. Wuest M, Morgenstern K, Graf EM, Braeter M, Hakenberg OW, Wirth MP, et al. Cholinergic and purinergic responses in isolated human detrusor in relation to age. J Urol. 2005;173:2182-9.

5. Kirschstein T, Protzel C, Porath K, Sellmann T, Köhling R, Hakenberg OW. Age-dependent contribution of Rho kinase in carbachol-induced contraction of human detrusor smooth muscle in vitro. Acta Pharmacol Sin. 2014;35:74-81

6. Ohl F, Jung M, Radonić A, Sachs M, Loening SA, Jung K. Identification and validation of suitable endogenous reference genes for gene expression studies of human bladder cancer. J Urol. 2006;175:1915-20.

7. Guo C, Liu S, Sun MZ. Novel insight into the role of GAPDH playing in tumor. Clin Transl Oncol. 2013;15:167-72.

8. Vajda A, Marignol L, Barrett C, et al. Gene expression analysis in prostate cancer: the importance of the endogenous control. Prostate. 2013;73:382-90.

9. Tang SW, Chang WH, Su YC, et al. MYC pathway is activated in clear cell renal cell carcinoma and essential for proliferation of clear cell renal cell carcinoma cells. Cancer Lett. 2009;273:35-43.

10. Ahmad SS, Glatzle J, Bajaeifer K, et al. Phosphoglycerate kinase 1 as a promoter of metastasis in colon cancer. Int J Oncol. 2013;43:586-90.

11. Zhao W, Chang C, Cui Y, et al. Steroid receptor coactivator-3 regulates glucose metabolism in bladder cancer cells through coactivation of hypoxia inducible factor 1a. J Biol Chem. 2014;289:11219-29.

12. Abe H, Kamai T, Hayashi K, Anzai N, Shirataki H, Mizuno T, et al. The Rho-kinase inhibitor HA-1077 suppresses proliferation/migration and induces apoptosis of urothelial cancer cells. BMC Cancer. 2014;14:412.

13. Wibberley A, Chen Z, Hu E, Hieble JP, Westfall TD. Expression and functional role of Rho-kinase in rat urinary bladder smooth muscle. Br J Pharmacol. 2003;138:757-66.
14. Uehata M, Ishizaki T, Satoh $H$, et al. Calcium sensitization of smooth muscle mediated by a Rho-associated protein kinase in hypertension. Nature. 1997;389:990-4.

15. Goodman KB, Cui H, Dowdell SE, et al. Development of dihydropyridone indazole amides as selective Rho-kinase inhibitors. J Med Chem. 2007;50:6-9.

16. Li XH, Peng J, Tan N, Wu WH, Li TT, Shi RZ, et al. Involvement of asymmetric dimethylarginine and Rho kinase in the vascular remodeling in monocrotaline-induced pulmonary hypertension. Vascul Pharmacol. 2010;53:223-9.

17. Do Z, Fukumoto Y, Takaki A, Tawara S, Ohashi J, Nakano M, et al. Evidence for Rho-kinase activation in patients with pulmonary arterial hypertension. Circ J. 2009;73:1731-9.

18. Rajasekaran M, Mehta N, Baquir A, Kuntz S. Rho-kinase inhibition suppresses potassium chloride-induced bladder hyperactivity in a rat model. Urology. 2007:69:791-4.

19. Bing W, Chang S, Hypolite JA, et al. Obstruction-induced changes in urinary bladder smooth muscle contractility: a role for Rho kinase. Am J Physiol Renal Physiol. 2003;285:F990-7.

20. Chang S, Hypolite JA, DiSanto ME, Changolkar A, Wein AJ, Chacko S. Increased basal phosphorylation of detrusor smooth muscle myosin in alloxan-induced diabetic rabbit is mediated by upregulation of Rho-kinase beta and CPI-17. Am J Physiol Renal Physiol. 2006;290:F650-6.

\section{Submit your next manuscript to BioMed Central and take full advantage of:}

- Convenient online submission

- Thorough peer review

- No space constraints or color figure charges

- Immediate publication on acceptance

- Inclusion in PubMed, CAS, Scopus and Google Scholar

- Research which is freely available for redistribution 